\title{
BMJ Open Prognostic factors for work disability in patients with chronic widespread pain and fibromyalgia: protocol for a cohort study
}

\author{
Pernille H Duhn (D) , ${ }^{1,2}$ Henning Locht, ${ }^{2}$ Eva Ejlersen Wæhrens, ${ }^{1,3}$ \\ Robin Christensen, ${ }^{1,4}$ Karsten Thielen, ${ }^{5}$ Marius Henriksen, ${ }^{1}$ Lars Erik Kristensen, ${ }^{1,2}$ \\ Henning Bliddal, ${ }^{1,6}$ Kirstine Amris ${ }^{1,2}$
}

To cite: Duhn PH, Locht $\mathrm{H}$, Wæhrens EE, et al. Prognostic factors for work disability in patients with chronic widespread pain and fibromyalgia: protocol for a cohort study. BMJ Open 2021;11:e052919. doi:10.1136/ bmjopen-2021-052919

- Prepublication history for this paper is available online. To view these files, please visit the journal online (http://dx.doi org/10.1136/bmjopen-2021 052919).

Received 28 April 2021 Accepted 24 November 2021

Check for updates

(c) Author(s) (or their employer(s)) 2021. Re-use permitted under CC BY-NC. No commercial re-use. See rights and permissions. Published by BMJ.

For numbered affiliations see end of article.

Correspondence to Dr Pernille H Duhn; pernille.hurup.duhn@regionh.dk

\section{ABSTRACT}

Introduction The association between chronic widespread pain (CWP) and disability is well established. Although research support large interindividual differences in functional outcomes, limited studies are available on the socio-economic consequences of offering stratified treatment based on prognostic factors. Identification of predictors of long-term functional outcomes such as work disability as a critical consequence, could assist early and targeted personalised interventions. The primary objective of this cohort study is to identify prognostic factors for the primary endpoint work status (employed and working vs not working) in patients with CWP assessed 3 years from baseline, that is, at referral for specialist care.

Methods and analyses Data are collected at the diagnostic unit at Department of Rheumatology, Frederiksberg Hospital. The first 1000 patients $\geq 18$ years of age registered in a clinical research database (DANFIB registry) with CWP either 'employed and working' or 'not working' will be enrolled. Participants must meet the American College of Rheumatology 1990 definition of CWP, that is, pain in all four body quadrants and axially for more than 3 months and are additionally screened for fulfilment of criteria for fibromyalgia. Clinical data and patient-reported outcomes are collected at referral (baseline) through clinical assessment and electronic questionnaires. Data on the primary endpoint work status at baseline and 3 years from baseline will be extracted from the Integrated Labour Market Database, Statistics Denmark and the nationwide Danish DREAM database. Prognostic factor analysis will be based on multivariable logistic regression modelling with the dichotomous work status as dependent variable.

Ethics and dissemination Sensitive personal data will be anonymised according to regulations by the Danish Data Protection Agency, and informed consent are obtained from all participants. Understanding and improving the prognosis of a health condition like CWP should be a priority in clinical research and practice. Results will be published in international peer-reviewed journals. Trial registration number NCT04862520.

\section{INTRODUCTION}

Chronic pain is associated with substantial economic burden for the healthcare system
Strengths and limitations of this study

- Potential prognostic factors for developing painrelated work disability can be identified in patients with chronic widespread pain (CWP).

- This study describes a large clinical cohort of patients with CWP.

- The study will add knowledge to the risk profiling of patients with CWP.

- Recruitment from a specialised tertiary care setting may limit generalisability of study findings.

and work force and it has been demonstrated that the costs tend to increase as the pain condition gradually limits the patient's functional ability. ${ }^{1}$ Chronic widespread pain (CWP) is prevalent in the background population with an estimated prevalence of about $10 \%$ and represents a major clinical challenge due to the complexity of the disease. ${ }^{23}$ Apart from pain and other centrally mediated symptoms, CWP is strongly associated with disability affecting activities of daily living (ADLs), incapacity for normal employment and poor social participation, and incurs high direct medical costs as well as significant indirect costs, for example, sick leave and disability compensation. ${ }^{4-6}$ Fibromyalgia (FM) is the best characterised subset of patients presenting with CWP and is by many considered to represent the upper end of a pain severity spectrum, that is, associated with greater disease burden and higher levels of disability, in comparison to patients with CWP not fulfilling FM disease criteria or more localised pain conditions. ${ }^{78}$ Only few studies have explored predictors of long-term functional outcomes including work disability in the CWP population and only few data are available on the socioeconomic benefits of intervention. ${ }^{9-11}$ In a study performed by 
our study group, participants' baseline intake of analgesics and pain phenotype were shown to predict observed functional ability as an outcome of a standardised rehabilitation programme 6 months postintervention. ${ }^{10}$ The following predictors of work disability in patients with FM have been identified in a longitudinal multicentre survey published in 1997: pain, self-reported functional ability, and unmarried status. ${ }^{12}$ Furthermore, in a Finish twin cohort study, the burden of FM-associated symptoms was shown to strongly predict early disability retirement. ${ }^{13}$ Work-related factors like heavy workload or low decision authority, previously identified as general risk factors for early disability retirement, had only marginal effect in this study. ${ }^{13}$ A systematic review discovered that treatment studies evaluating work disability as outcome in FM were scarce. ${ }^{14}$ The conclusion was that more studies of treatment effects on outcomes related to work disability and longitudinal studies to explore long-term effects of symptoms on work disability were needed. ${ }^{14}$ By quantifying a significant health-related at-work productivity loss as a critical outcome it is anticipated that major stakeholders-such as policy-makers-will get insights as to the resource use and thus the economic impact of CWP on both society and individual.

\section{Rationale and hypothesis}

Prognostic factor research aims to discover and evaluate factors that might be useful as modifiable targets for interventions or predictors of differential treatment responses. ${ }^{15} 16$ It is a fundamental component of 'stratified medicine', which refers to the targeting of pharmacological and non-pharmacological interventions according to the biological or clinical risk characteristics shared by subgroups of patients and generally contextual factors in rheumatology. ${ }^{17-19}$ By using prognostic factor research, the purpose is to understand and improve future outcomes in patients with CWP. Identification of prognostic factors for long-term functional outcomes, including work disability, could assist tailoring and timing the therapeutic decision for specific patients based on risk profiling and potentially optimise functional outcomes compared with offering standardised (ie, ignorant) intervention programmes to patients with CWP.

\section{Objectives}

Our aim is to understand and improve future outcomes in people with CWP. Thus, this study has three objectives:

I. To reveal prognostic factors, among the cohort of CWP patients, that are associated with the primary endpoint work status (employed and working vs not working) assessed after 3 years.

II. Identification of prognostic factors for work status (employed and working vs not working) after 3 years in patients with CWP, who are employed and working at baseline.

III. Identification of prognostic factors for work status (employed and working vs not working) after 3 years in patients with CWP, who are not working at baseline.

The potential prognostic factors for variation in work status will be explored among the following covariates:

1. Age.

2. Sex.

3. Level of education.

4. Marital status.

5. Symptom duration.

6. Primary CWP (no other established rheumatic disease).

7. Secondary CWP (as comorbidity to other established rheumatic disease).

8. Number of weeks outside the labour market the preceeding 5 years.

9. Labour market affiliation and work status

- Employed and working.

- Not working.

- Interdisciplinary rehabilitation programme.

10. Baseline use of analgesics

11. Self-reported functional ability and overall symptom burden assessed by the functioning and symptom subscales of the Revised Fibromyalgia Impact Questionnaire (FIQR).

12. Self-reported ability to cope with pain assessed by the Pain Self Efficacy Questionnaire (PSEQ).

13. Pain phenotype: tender point (TP) count, total score on the Pain Detect Questionnaire (PDQ), total score on the Widespread Pain Index (WPI).

14. Fulfilment of criteria for FM in addition to CWP.

The reason for considering these covariates is based on previous studies, as mentioned in the introduction section; work disability in FM has been associated with level of pain, self-reported functional ability and unmarried status. ${ }^{12}{ }^{14}$ Intake of analgesics and pain phenotype has been shown to predict observed functional ability. ${ }^{10}$ The overall burden of FM-associated symptoms has been found to be a predictor of loss of employment and early disability retirement. ${ }^{131420} \mathrm{FM}$ patients who are employed and working report better health, and patients with a pending social welfare application perform worse than the ones without. ${ }^{2021}$ Finally, the social and motivational support from family seem to enable the maintenance of work for women with FM. ${ }^{14}$

\section{METHODS AND ANALYSIS}

\section{Study design}

The study is designed as a clinical cohort study enabling multivariable logistic regression modelling of data from the large Danish Fibromyalgia registry (DANFIB) cohort of CWP patients with the primary aim assessed after 3 years.

\section{Patient and public involvement}

This project follows the European League Against Rheumatism recommendations for the inclusion of patient representatives in contemporary scientific projects. ${ }^{22}$ 
The cohort study is designed with assistance from two Danish patient representatives, Trine Leth (TL) and Elena Andersen (EA). Both TL and EA are diagnosed with FM and were invited while participating in a 2-week group-based rehabilitation programme at Department of Rheumatology, Frederiksberg Hospital. They have participated in discussions of relevance to the cohort study, but not the recruitment of participants. The author group will disseminate results to the participants through The Danish Fibromyalgia Association, in which there is a good collaboration.

\section{Setting}

Data collection takes place in a specialised clinical care setting at the diagnostic unit at Department of Rheumatology, Frederiksberg Hospital. Here patients presenting with CWP, either as their primary pain problem or secondary to other established rheumatic disease, have been offered clinical assessment and screening for CWP since 1 January 2018.

The diagnostic work-up includes manual TP examination and an electronic questionnaire based multidimensional assessment (table 1). The electronic data collection is accessed via touchscreens and data are exported to a designated clinical research database (the DANFIB registry). The use of computerised health status questionnaires in FM populations has prior been validated by the Parker Institute. ${ }^{23}$ Data extracted from electronic patient files, including findings at clinical examination (manual TP count), are also integrated into the DANFIB registry. A protocol outlining the content and objectives of the DANFIB registry can be accessed online. ${ }^{24}$ The DANFIB registry will serve as point of departure for the current cohort study.

\section{Participants}

The first 1000 patients consecutively registered in theDANFIB registry with CWP independent of working status (figure 1). Participant inclusion is expected to be completed by December 2021. Informed consent will be obtained for all participants when registered in the DANFIB registry. To be eligible for enrolment, participants must be over 18 years of age, able to understand and read Danish, and have retrievable data in the DANFIB registry. Participants at baseline receiving pension, that is, public or early retirement pension, disability pension or retirement will be excluded, because their work status is resolved. Thus, participants will be excluded from the study if the following criteria are present: (1) no consent, (2) do not read and understand Danish, (3) nonretrievable data in the DANFIB registry and (4) pension.

\section{Variables and outcome measures}

\section{Data sources}

Baseline demographics, clinical characteristics and individual labour market status assessed at baseline will be extracted from the DANFIB registrytable 1). Data on the primary endpoint work status ('employed
Table 1 Summary of data to be collected

\begin{tabular}{|c|c|c|}
\hline & Baseline & 3 years \\
\hline \multicolumn{3}{|l|}{ Baseline demographic } \\
\hline $\operatorname{Sex}(M / F)$ & $\mathrm{X}$ & - \\
\hline Age (years) & $x$ & - \\
\hline Labour market affiliation & $x$ & - \\
\hline Duration of pain (weeks) & $x$ & - \\
\hline $\begin{array}{l}\text { Level of education (primary or high school, } \\
\text { medium-term or long higher education) }\end{array}$ & $x$ & - \\
\hline $\begin{array}{l}\text { Marital status (married, cohabiting, } \\
\text { separated/divorced, widowed, single) }\end{array}$ & $x$ & - \\
\hline $\begin{array}{l}\text { Outcome of clinical assessment (pain } \\
\text { diagnosis) }\end{array}$ & $x$ & - \\
\hline $\begin{array}{l}\text { Other inflammatory or degenerative } \\
\text { rheumatic diseases }(y / n)\end{array}$ & $x$ & - \\
\hline \multicolumn{3}{|l|}{ Baseline analgesics: } \\
\hline $\begin{array}{l}\text { Use of mild analgesics, including NSAIDs } \\
(y / n)\end{array}$ & $x$ & - \\
\hline Use of anti-rheumatics $(y / n)$ & $x$ & - \\
\hline $\begin{array}{l}\text { Use of antidepressant for example, } \\
\text { Amitriptyline }(y / n)\end{array}$ & $x$ & - \\
\hline $\begin{array}{l}\text { Use of antiepileptics, for example, } \\
\text { Gabapentin }(y / n)\end{array}$ & $x$ & - \\
\hline $\begin{array}{l}\text { Use of muscle relaxants for example, } \\
\text { Chlorzoxazone }(y / n)\end{array}$ & $x$ & - \\
\hline Low dose naltrexone $(y / n)$ & $x$ & - \\
\hline Use of Cannabinoids $(y / n)$ & $x$ & - \\
\hline \multicolumn{3}{|l|}{ Clinical examination: } \\
\hline Tender point count $(0-18)$ & $x$ & - \\
\hline \multicolumn{3}{|l|}{ Patient-reported outcomes: } \\
\hline FIQR & $x$ & - \\
\hline PSEQ & $x$ & - \\
\hline $\mathrm{PDQ}$ & $x$ & - \\
\hline SSS & $x$ & - \\
\hline WPI & $x$ & - \\
\hline \multicolumn{3}{|l|}{ Official registryextraction: } \\
\hline DREAM database & $x$ & $x$ \\
\hline Integrated Labour Market Database & $\mathrm{x}$ & $x$ \\
\hline
\end{tabular}

FIQR, Fibromyalgia Impact Questionnaire; M/F, Male/Female; PDQ, Pain Detect Questionnaire; PSEQ, Pain Self Efficacy Questionnaire; SSS, Symptom Severity Scale; y/n, yes/no; WPI, Widespread Pain Index.

and working' or 'not working') at baseline and again 3 years from baseline will be extracted from the Integrated Laboure Market Database at Statistics Denmark and the nationwide Danish DREAM database.

\section{Measurements}

Baseline demographics and clinical characteristics

Data on age and sex are based on the Danish Civil Registration (CPR) number, given at birth and unique to every Danish citizen. Data concerning labour market status (ensuring sufficient enrolment), level of education, marital status, primary or secondary CWP, symptom 


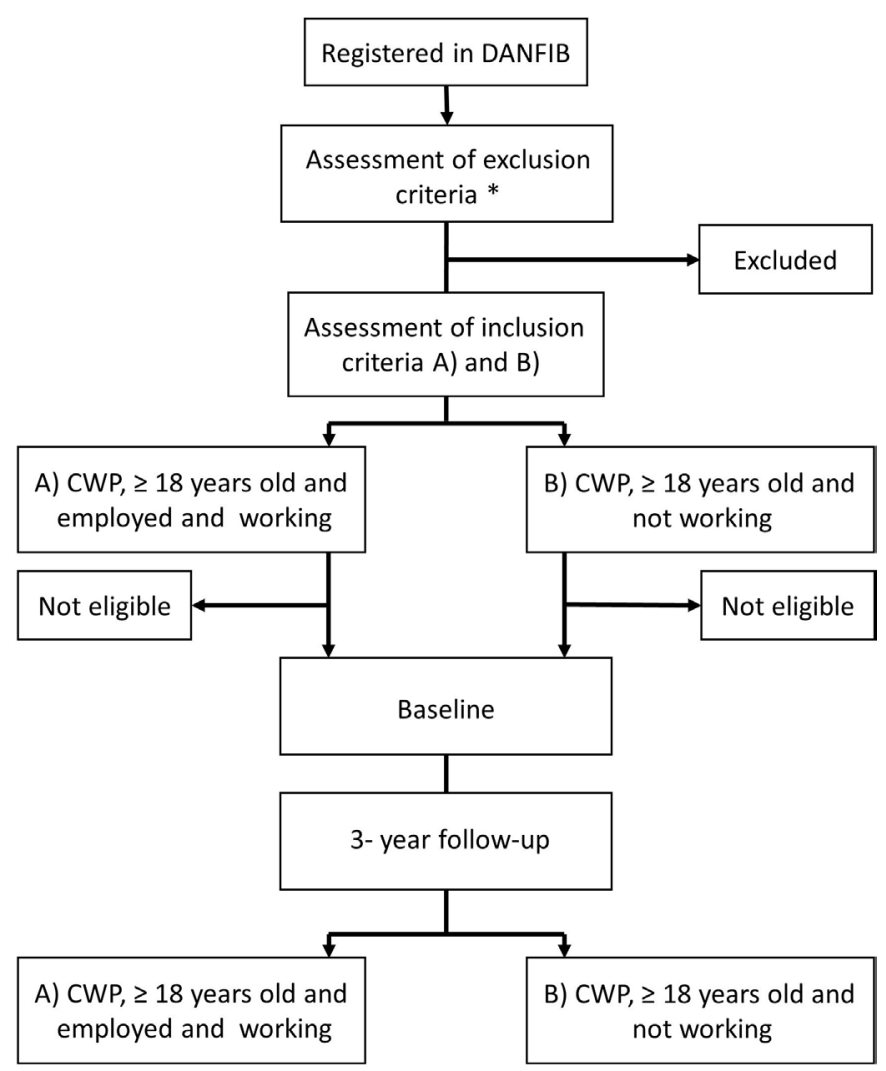

Figure 1 Overview of participant flow. *Exclusion criteria: (1) no consent, (2) do not read and understand Danish, (3) nonretrievable data in the DANFIB and (4) pension. CWP, chronic widespread pain.

duration, baseline use of analgesics, and scores obtained by the FIQR, PSEQ, PDQ, Symptom Severity Scale (SSS) and WPI are self-reported by the patient, based on the following patient-reported outcome measurements (PROMs):

1. The FIQR, which is the recommended self-rating tool in evaluating disease burden and impact of disease on ADLs in patients with FM. ${ }^{25}$ The FIQR consists of 21 individual questions categorised in three different domains termed; function (ADL), overall impact and symptoms ${ }^{26}$ The domain 'function' includes nine items, 'overall impact' two items and 'symptoms' ten items. ${ }^{26}$ All items are rated on an 11-category numeric rating scale $(0-10)$, with 10 representing the 'worst' scenario (eg, very difficult, no energy, very tender). ${ }^{26}$ All questions refer to the context of the past 7 days. ${ }^{26}$ The scoring of the FIQR is a summed score for 'function' (range 0-90) divided by three with a max score of 30, a summed score for 'impact' (range 0-20) with a max score of 20 and a summed score for 'symptoms' (range $0-100$ ) divided by two with a max score of $50 .{ }^{26}$ The three domain scores are summed into a FIQR total score. ${ }^{26}$

2. The PSEQ originally developed and validated in a heterogeneous group of patients with chronic pain and patients with low back pain. ${ }^{27}$ The PSEQ has been translated, culturally adapted and validated in a Dan- ish population of FM patients. ${ }^{28}$ The PSEQ consists of ten items reflecting a variety of ADL tasks and life situations, including paid work. Patients are asked to rate their perceived confidence in performing tasks while in pain on a 0-6 Numeric Rating Scale with a max score of 60 . The higher the score, the greater selfefficacy. ${ }^{27} 28$

3. The PDQ, originally developed and validated in 2006 for the purpose of establishing a screening questionnaire to detect the likelihood of a neuropathic pain component in patients with low back pain. ${ }^{29}$ Since then the PDQ has proved to be a useful instrument in identification of neuropathic pain features in various clinical conditions including CWP. ${ }^{30-32}$ The PDQ consists of seven items which are somatosensory descriptor items rated on a 0-5 Numeric Rating Scale: never, hardly noticed, slightly, moderately, strongly and very strongly. Questions regarding pain intensity on a VAS scale), the radiating quality of the pain (yes/no) and the course of pain (selection between four pain course patterns). For screening purposes, the PDQ has a score ranging from -1 to 38 with cut-off scores $\leq 12$ (a neuropathic pain component is unlikely) and $\geq 19$ (a neuropathic pain component is likely), respectively. ${ }^{29}$

4. The SSS and WPI, which constitutes part of the 2016 American College of Rheumatology (ACR) diagnostic criteria for FM. ${ }^{33}$ The SSS sums the 0-3 scores of major defining symptoms, including waking unrefreshed, dyscognition and fatigue with a resulting sum score ranging from 0 to 12 . The WPI divides the body into 19 body sites and five regions and the WPI score indicates in which of the 19 predefined pain sites there have been pain during the last week, corresponding to a WPI score ranging between 0 and 19. The ACR 2016 diagnostic criteria for FM are met if: (WPI $\geq 7$ and SSS $\geq 5$ ) or (WPI 4-6 and SSS $\geq 9$ ). Furthermore, a generalised pain criterion must be satisfied, defined as pain in minimum four of five regions. ${ }^{33}$

\section{Clinical examination}

The manual TP count and fulfilment of criteria for FM is assessed at baseline. The clinical examination comprises a manual TP examination according to 1990 ACR guidelines $^{34}$ (table 1). The 1990 ACR definition of CWP are considered met if the patient present with persistent or recurrent pain during the last 3 months located both axially plus in all four body quadrants, whereas FM patients present with CWP according to the 1990 ACR definition and a minimum of $11 / 18 \mathrm{TP}$, that is, fulfils the dual 1990 ACR classification criteria and/or fulfil the ACR 2016 diagnostic criteria. ${ }^{33} 34$

\section{Register-based data on participants' work status}

Data collection regarding the primary endpoint on participants' work status at baseline and 3 years from baseline will be based on the CPR number, which makes it possible to link the DANFIB registry with other official registry data on employment, occupation and income. Data on 


\section{Box 1 Primary endpoint and labour market affiliation}

Work status
Working
Employed
Self-employed
Flexjob*
Under education
Part-time sick leave $†$
Not working
Unemployed
Social assistance, activated and non-activatedc $\ddagger$
Interdisciplinary rehabilitation programmed§
Sick leave
Supported by a spouse, home maker
*Subsidised job scheme, created for persons with limited work capacity.
†People who are employed and working part time.
†Transfer income administered by the municipal social service department.
§Rehabilitation programme offered to people with realistic return to work
prospective.

employment status will be extracted from the Integrated Labour Market Database, Statistics Denmark. Employers report data on their employees to Statistics Denmark each year in November. ${ }^{35}$ Work status will be collected from all participants the preceeding 5 years before baseline, at baseline and again 3 years from baseline to ensure the accuracy of data as opposed to PROMs.

Data on participants receiving social transfer payment will be extracted through the nationwide Danish DREAM database. ${ }^{36}$ The DREAM database is based on data from the Danish Ministry of Employment, Ministry of Social Affairs, Ministry of Education, Ministry of Integration, Statistics Denmark and the 98 municipalities. DREAM contains data on all the Danish citizens receiving social benefits or any other transfer income. Information is obtained prospectively each week. Furthermore, Statistics Denmark has a database that compares every person's job with their job the previous year and defines the work status on that basis, which includes a change of job position and workplace, as well as mobility in or out of unemployment. Labour market affiliation at baseline and 3 years after baseline will be categorised as 'working' and 'not working' as illustrated in box 1. Those 'working' will include participants that are employed, self-employed or in a 'flexjob' and working. A rehabilitation team in each municipality assigns people to a 'flexjob', interdisciplinary rehabilitation programme, disability pension or another plan. 'Flexjob' is a subsidised job scheme, created for persons with limited work capacity, consequently the person receives ordinary wage paid by the employer and a supplemental social benefit which is paid by municipality. Participants undergoing a formal education or training will be presumed able to work and considered as employed. Whereas 'not working' will cover participants receiving transfer payment benefits including unemployment benefits, social assistance, full sick leave, an interdisciplinary rehabilitation programme, support by a spouse and home makers. If a person is on sick leave, a municipally administered benefit is paid to the person or transferred to the employer, if the employer pays the ordinary wage to the sick-listed employee. In Denmark, getting sick- leave payment benefits, might refer to persons which are still employed or unemployed. A person can have a part-time sick leave and thereby still by employed and working part-time. In this cohort study, those on part-time sick leave will be considered as 'working'. The interdisciplinary rehabilitation programme refers to people, who are not able to work and comprises activities based on recommendations from the rehabilitation team. The programme consists of health education and employment opportunities and last from 1 to 5 years. The interdisciplinary rehabilitation programme is offered to people, whom the rehabilitation team evaluated with realistic return to work prospective and are therefore of particular interest in the context of patients with CWP.

\section{Bias and confounding}

Selection of the participants reflects recruitment from a specialised tertiary care setting, but participants selected for the primary endpoint work status (employed and working vs not working) was done using rigorous criteria to avoid ambiguous results. Recall bias could occur for the participants that is, regarding current medication and other patient-reported data. Attrition bias can occur if there is an unequal lost to follow-up between the prognostic factor groups. In clinical trials, participants might withdraw due to unsatisfactory treatment efficacy, intolerable adverse events or even death.

The 14 different covariates will all be considered as potential prognostic factors for variation in work status and could also be regarded as confounders. The challenge with observational data is that prognostic 'exposure groups' are not applied randomly, possibly leading to selection bias and confounding variables. Consequently, it is sensible to try to estimate the 'causal effects' of prognostic factors. Propensity score methods are reliable tools for addressing such objectives because the assumptions needed to make their answers appropriate are more assessable and transparent to the investigator. ${ }^{37}$ Improved confounding variable balance between groups will be achieved by matching observations from each prognostic group based on the propensity score.$^{38}$ Thus, by use of propensity score methods attempt will be made to correct for the 'assignment mechanism' by finding unexposed units similar to exposure units $\left(Y_{0}\left|X_{\mathrm{E}} \approx Y_{0}\right| X_{\mathrm{U}}\right)$. The following pragmatic definition of what makes a confounding variable $(C)$ will be used:

- The covariate $(C)$ is an ancestor (cause) of the outcome $(Y)$.

- The covariate $(C)$ probably cause the exposure $(X$; for example, group).

- The covariate $(C)$ is not a descendant (effect) of the exposure $(X)$ or outcome $(Y)$. 


\section{Statistical analyses}

\section{Sample size considerations}

The clinical cohort in this study will comprise the first 1000 consecutively enrolled patients in the DANFIB registry either 'employed and working' or 'not working', given the PROMs on labour market status. For a comparison of two independent binomial proportions (comparing prognostic factor exposed vs unexposed) using Pearson's $\chi^{2}$ statistic with a $\chi^{2}$ approximation with a two-sided significance level of 0.05 , a sample size of 388 per group (776 in total) achieves a power of at least $80 \%$; when comparing $50 \%$ vs $40 \%$.

\section{Descriptive statistics and main analyses}

All descriptive statistics and tests will be reported in concurrence with the recommendations of the 'Enhancing the QUAlity and Transparency Of health Research' network: the Strengthening the Reporting of Observational Studies in Epidemiology Statement. ${ }^{39-41}$ Crude and adjusted estimates will be reported. All analyses will be carried out using R software V.3.6.2 (2019-12-12). Baseline variables will be described for all participants. Continuous data and ordinal scales will be reported descriptively using Means and SDs or medians and IQRs depending on data distribution. Dichotomous data will be reported as an absolute number as well as the relative number (\%).

Analysis population: The primary analyses will be based on the Intention to Survey (ITS) population, that is, based on the first 1000 patients enrolled in the cohort study. The ITS principle asserts the effect of being enrolled in the DANFIB cohort study (that is, the planned survey regimen), rather than the actual survey given (ie, it is independent of survey adherence), irrespective of their adherence to the planned course of survey (ie, independent of drop-outs).

All 95\% CIs and p values will be two sided. Explicit adjustments for multiplicity will not be applied, instead the Statistical Analysis Plan will prespecify how the secondary and tertiary objectives will be analysed in a prioritised order (eg, 'gatekeeping procedure'). ${ }^{42}$

Analysis model: The prognostic value will be studied through a (multivariable) logistic regression model also calculating the OR. The primary outcome will be the dichotomous work status ('employed and working' ws 'not working' according to box 1) in participants with CWP at baseline and 3 years from baseline. The coefficients associated with each potential prognostic factor will be estimated.

Missing data: Every effort will be made to minimise missing outcome data. Multiple imputation will be used to account for participants who have the measurement at baseline but are missing the outcome at follow-up, for example, due to maternity leave, emigration or death. No adjustment is expected in the analysis regarding missing data for any of the 14 covariates (age, sex, level of education, etc).

Sensitivity analyses: Four explicit sensitivity analyses for the primary aim will be performed to assess the strength of the results and to account for missing data. The first sensitivity analysis involves specifying a pessimistic imputation model (all participants are not working), the second sensitivity analysis involves specifying an optimistic imputation model (all participants are working), the third sensitivity analysis involve reanalysis of the primary aim using data from the 'per-protocol' population, and finally the fourth analysis set will be based on the 'Data As Observed' population.

\section{DISCUSSION}

Work disability is a serious concern in patients with CWP at both the individual and societal level. It is reported that among FM patients encountered in tertiary care settings, only about one third are part of the work force at the time of referral. ${ }^{21}$ Early intervention and individual adjustments in the work situation matching the level of ability may improve retention in employment. ${ }^{43}$ Acknowledging the substantial negative impact of CWP on the individual's capacity for normal employment, the proposed cohort study aims to identify prognostic factors for the development of work disability and unemployment in this patient population. The results from this cohort study are anticipated to contribute with relevant knowledge that may be used to guide future intervention matching and delivery of stratified interventions based on prognostic classification.

\section{Ethics and dissemination}

The DANFIB registry has been approved by the Danish Data Protection Agency and granted authorisation for the period January 2018 to January 2033 (j.nr.: 201258-0004). The cohort study is approved by the regional scientific ethical committee (j.nr: P-2020-967). Sensitive personal data will be anonymised according to regulations stipulated by the Danish Data Protection Agency, and informed consent are obtained from all patients before enrolment in the DANFIB registry. The registry data analysed at Statistics Denmark will replace the original CPR numbers with other personal identification numbers in order to make the people in the dataset anonymous to the researchers. Consequently, the project does not require approval from the Danish Data Protection Agency. Papers will be submitted for publication in international peerreviewed journals. Results will also be presented orally and as posters at international conferences and at the Danish Society of Rheumatology's annual meetings.

\section{Author affiliations}

${ }^{1}$ Parker Institute, Frederiksberg Hospital, Frederiksberg, Denmark ${ }^{2}$ Rheumatology, Frederiksberg Hospital, Frederiksberg, Denmark

${ }^{3}$ Occupational Science \& Occupational Therapy, User Perspectives and CommunityBased Research, Department of Public Health, University of Southern Denmark, Odense, Denmark

${ }^{4}$ Research Unit of Rheumatology, Department of Clinical Research, University of Southern Denmark, Odense, Denmark

${ }^{5}$ Department of Social Medicine, University of Copenhagen, Kobenhavn, Denmark ${ }^{6}$ Clinical Medicine, University of Copenhagen, Kobenhavn, Denmark 
Acknowledgements The authors thank the patient representatives Trine Leth (TL) and Elena Andersen (EA) for taking part in the process of preparing the current cohort study and Christian Cato for consultancy regarding the DANFIB registry.

Contributors KA is research coordinator and daily manager of the DANFIB registry. PHD is the principal investigator for this clinical cohort study. KA, PHD, RC, MH and KT will be responsible for data extraction and data analysis. PHD, HL, EEW, RC, LEK, $\mathrm{HB}, \mathrm{MH}, \mathrm{KT}$ and $\mathrm{KA}$ participated in the design of the cohort study and drafting of the protocol. All authors have given final approval for the protocol to be published.

Funding This work was supported by Minister Erna Hamilton foundation (J.nr. 21-2020), The Danish Rheumatism Association (R167-A5693) and The Fond of Bispebjerg and Frederiksberg Hospital (no funding number available). The Parker Institute, Bispebjerg and Frederiksberg Hospital is supported by a core grant from the Oak Foundation (OCAY-18-774-OFIL).

Competing interests MH: Advisory Board member at the Thuasne Group. LEK: received fees for speaking and/or consultancy from Pfizer, AbbVie, Amgen, UCB, Celgene, BMS, Biogen, Sanofi, MSD, Novartis, Eli Lilly, Janssen Pharmaceuticals. PHD, HL, EEW, RC, KT, HB and KA: none.

Patient and public involvement Patients and/or the public were involved in the design, or conduct, or reporting, or dissemination plans of this research. Refer to the Methods section for further details.

Patient consent for publication Not applicable.

Provenance and peer review Not commissioned; externally peer reviewed.

Open access This is an open access article distributed in accordance with the Creative Commons Attribution Non Commercial (CC BY-NC 4.0) license, which permits others to distribute, remix, adapt, build upon this work non-commercially, and license their derivative works on different terms, provided the original work is properly cited, appropriate credit is given, any changes made indicated, and the use is non-commercial. See: http://creativecommons.org/licenses/by-nc/4.0/.

\section{ORCID iD}

Pernille H Duhn http://orcid.org/0000-0001-8729-0634

\section{REFERENCES}

1 Lalonde L, Choinière M, Martin $\mathrm{E}$, et al. Costs of moderate to severe chronic pain in primary care patients - a study of the ACCORD Program. J Pain Res 2014;7:389-403.

2 Creed F. A review of the incidence and risk factors for fibromyalgia and chronic widespread pain in population-based studies. Pain 2020;161:1169-76.

3 Mansfield KE, Sim J, Jordan JL, et al. A systematic review and metaanalysis of the prevalence of chronic widespread pain in the general population. Pain 2016;157:55-64.

4 Mills SEE, Nicolson KP, Smith BH. Chronic pain: a review of its epidemiology and associated factors in population-based studies. $\mathrm{Br}$ $J$ Anaesth 2019;123:e273-83.

5 Wæhrens EE, Amris K, Fisher AG. Performance-based assessment of activities of daily living (ADL) ability among women with chronic widespread pain. Pain 2010;150:535-41.

6 Wæhrens EE, Bliddal H, Danneskiold-Samsøe B, et al. Differences between questionnaire- and interview-based measures of activities of daily living (ADL) ability and their association with observed ADL ability in women with rheumatoid arthritis, knee osteoarthritis, and fibromyalgia. Scand J Rheumatol 2012;41:95-102.

7 Schaefer C, Mann R, Masters ET, et al. The comparative burden of chronic widespread pain and fibromyalgia in the United States. Pain Pract 2016;16:565-79.

8 Amris K, Wæhrens EE, Jespersen A, et al. Observation-based assessment of functional ability in patients with chronic widespread pain: a cross-sectional study. Pain 2011;152:2470-6.

9 Häuser W, Bernardy K, Arnold B, et al. Efficacy of multicomponent treatment in fibromyalgia syndrome: a meta-analysis of randomized controlled clinical trials. Arthritis Rheum 2009;61:216-24.

10 Amris K, Luta G, Christensen R, et al. Predictors of improvement in observed functional ability in patients with fibromyalgia as an outcome of rehabilitation. J Rehabil Med 2016;48:65-71.

11 de Rooij A, Roorda LD, Otten RHJ, et al. Predictors of multidisciplinary treatment outcome in fibromyalgia:a systematic review. Disabil Rehabil 2013;35:437-49.

12 Wolfe F, Anderson J, Harkness D, et al. Work and disability status of persons with fibromyalgia. J Rheumatol 1997;24:1171-8.

13 Markkula R, Kalso E, Huunan-Seppälä A, et al. The burden of symptoms predicts early retirement: a twin cohort study on fibromyalgia-associated symptoms. Eur J Pain 2011;15:741-7.
14 Palstam A, Mannerkorpi K. Work ability in fibromyalgia: an update in the 21st century. Curr Rheumatol Rev 2017;13:180-7.

15 Riley RD, Hayden JA, Steyerberg EW, et al. Prognosis research strategy (progress) 2: prognostic factor research. PLoS Med 2013;10:e1001380.

16 Hemingway $\mathrm{H}$, Croft $\mathrm{P}$, Perel $\mathrm{P}$, et al. Prognosis research strategy (progress) 1: a framework for researching clinical outcomes. BMJ 2013;346:e5595-11.

17 Nielsen SM, Tugwell P, de Wit MPT, et al. Identifying provisional generic contextual factor domains for clinical trials in rheumatology: results from an OMERACT initiative. J Rheumatol 2019;46:1159-63.

18 Nielsen SM, Uggen Rasmussen M, Boers M, et al. Towards consensus in defining and handling contextual factors within rheumatology trials: an initial qualitative study from an OMERACT Working group. Ann Rheum Dis 2021;80:1-8.

19 Hingorani AD, Windt DAvander, Riley RD, et al. Prognosis research strategy (progress) 4: stratified medicine research. BMJ 2013;346:e5793-9.

20 Palstam A, Bjersing JL, Mannerkorpi K. Which aspects of health differ between working and nonworking women with fibromyalgia? A cross-sectional study of work status and health. BMC Public Health 2012;12:1076.

21 Amris K, Wæhrens EE, Christensen R, et al. Interdisciplinary rehabilitation of patients with chronic widespread pain: primary endpoint of the randomized, nonblinded, parallel-group improve trial. Pain 2014;155:1356-64.

22 de Wit MPT, Berlo SE, Aanerud GJ, et al. European League against rheumatism recommendations for the inclusion of patient representatives in scientific projects. Ann Rheum Dis 2011;70:722-6.

23 Wæhrens EE, Amris K, Bartels EM, et al. Agreement between touchscreen and paper-based patient-reported outcomes for patients with fibromyalgia: a randomized cross-over reproducibility study. Scand J Rheumatol 2015;44:503-10.

24 Amris K. DANFIB protocol [Internet], 2017. Available: http://www. parkerinst.dk/sites/default/files/danfib_protocol.pdf [Accessed 27 Jun 2020].

25 Boomershine CS. A comprehensive evaluation of standardized assessment tools in the diagnosis of fibromyalgia and in the assessment of fibromyalgia severity. Pain Res Treat 2012;2012:1-11.

26 Bennett RM, Friend R, Jones KD, et al. The revised fibromyalgia impact questionnaire (FIQR): validation and psychometric properties. Arthritis Res Ther 2009;11:415-14.

27 Nicholas MK. The pain self-efficacy questionnaire: taking pain into account. Eur J Pain 2007;11:153-63.

28 Rasmussen MU, Rydahl-Hansen S, Amris K, et al. The adaptation of a Danish version of the pain self-efficacy questionnaire: reliability and construct validity in a population of patients with fibromyalgia in Denmark. Scand J Caring Sci 2016;30:202-10.

29 Freynhagen R, Baron R, Gockel U, et al. painDETECT: a new screening questionnaire to identify neuropathic components in patients with back pain. Curr Med Res Opin 2006;22:1911-20.

30 Freynhagen R, Tölle TR, Gockel U, et al. The painDETECT project far more than a screening tool on neuropathic pain. Curr Med Res Opin 2016;32:1033-57.

31 Amris K, Jespersen A, Bliddal H. Self-Reported somatosensory symptoms of neuropathic pain in fibromyalgia and chronic widespread pain correlate with tender point count and pressure-pain thresholds. Pain 2010;151:664-9.

32 Rifbjerg-Madsen S, Wæhrens EE, Danneskiold-Samsøe B, et al. Psychometric properties of the paindetect questionnaire in rheumatoid arthritis, psoriatic arthritis and spondyloarthritis: Rasch analysis and test-retest reliability. Health Qual Life Outcomes 2017;15:1-12.

33 Wolfe F, Clauw DJ, Fitzcharles M-A, et al. 2016 revisions to the 2010/2011 fibromyalgia diagnostic criteria. Semin Arthritis Rheum 2016;46:319-29.

34 Wolfe F, Smythe HA, Yunus MB, et al. The American College of rheumatology 1990 criteria for the classification of fibromyalgia. Report of the multicenter criteria Committee. Arthritis Rheum 1990;33:160-72.

35 Hougaard Charlotte Ørsted, Nygaard E, Holm AL, et al. Job mobility and health in the Danish workforce. Scand J Public Health 2017;45:57-63.

36 Hjollund $\mathrm{NH}$, Larsen FB, Andersen JH. Register-based follow-up of social benefits and other transfer payments: accuracy and degree of completeness in a Danish interdepartmental administrative database compared with a population-based survey. Scand J Public Health 2007;35:497-502.

37 Rubin DB. Estimating causal effects from large data sets using propensity scores. Ann Intern Med 1997;127:757-63. 
38 Agoritsas T, Merglen A, Shah ND. Adjusted Analyses in Studies Addressing Therapy and Harm: Users' Guides to the Medical Literature. J Am Med Assoc 2017;317:748-59.

39 Altman DG, Simera I, Hoey J, et al. EQUATOR: reporting guidelines for health research. Lancet 2008;371:1149-50.

40 Christensen R, Bliddal H, Henriksen M. Enhancing the reporting and transparency of rheumatology research: a guide to reporting guidelines. Arthritis Res Ther 2013;15:109-4.
41 Vandenbroucke JP, von Elm E, Altman DG, et al. Strengthening the reporting of observational studies in epidemiology (STROBE): explanation and elaboration. PLoS Med 2007;4:e297-54.

42 Yadav K, Lewis RJ. Gatekeeping strategies for avoiding falsepositive results in clinical trials with many comparisons. JAMA 2017;318:1385-6.

43 Henriksson C, Liedberg G. Factors of importance for work disability in women with fibromyalgia. J Rheumatol 2000;27:1271-6. 